\title{
PRIME NUMBERS OF THE FORM $\left[n^{c}\right]$
}

\author{
J. RIVAT and J. WU \\ Institut Elie Cartan, UMR 7502 UHP-CNRS-INRIA, Université Henri Poincaré (Nancy 1), \\ 54506 Vandouvre-lès-Nancy, France \\ e-mail: rivat@iecn.u-nancy.fr,wujie@iecn.u-nancy.fr
}

(Received 4 October, 1999; revised 10 March, 2000)

\begin{abstract}
In 1953, Pjateckî-S̆apiro has proved that there are infinitely many primes of the form $\left[n^{c}\right]$ for $1<c<\frac{12}{11}$ (with an asymptotic result). This range, which measures our progress in the technique of exponential sums, has been improved by many authors. In this paper we obtain $1<c \leq \frac{243}{205}$.
\end{abstract}

1991 Mathematics Subject Classification. Primary 11L07. Secondary 11N25, $11 \mathrm{~N} 45$.

1. Introduction. One of the most odd problems in prime number theory is to represent infinitely many prime numbers by polynomials. Though this problem has been completely solved for linear polynomials by the Dirichlet theorem on primes in arithmetic progressions, until now it remains open for polynomials of degree greater than 1 and it seems to be extremely difficult. Iwaniec [6] has proved that there exists an infinity of integers $n$ such that $n^{2}+1$ has at most two prime factors. As another direction of approaching this problem, Pjatecki1-Šapiro [9] has proposed to investigate the prime numbers of the form $\left[n^{c}\right]$, where $c>1$ and $[t]$ denotes the integer part of $t$. Clearly $\left[n^{c}\right]$ can be regarded as "polynomials of degree $c$ ". Define

$$
\pi_{c}(x):=\mid\left\{n \leq x:\left[n^{c}\right] \text { is prime number }\right\} \mid .
$$

Pjateckï-S̆apiro [9] has shown for $1<c<\frac{12}{11}=1 . \overline{09}$ that

$$
\pi_{c}(x) \sim x / c \log x \quad(x \rightarrow \infty) .
$$

This range has been improved by many authors. A detailed history can be found in [10]. The best record known is due to Rivat and Sargos [11]: $1<c<\frac{2817}{2426} \approx 1.16117$. Jia and Rivat have proved, independently, that

$$
\pi_{c}(x) \gg x / c \log x \quad\left(x \geq x_{0}\right)
$$

for $1<c<\frac{7}{6}=1.1 \overline{6}$ (Rivat [10]) and $1<c \leq \frac{20}{17} \approx 1.17647$ (Jia [6, I], and independently Baker, Harman and Rivat [1]). This result has been improved to $1<c<\frac{13}{11}=1 . \overline{18}$ by Jia [6, II] and to $1<c \leq \frac{45}{38} \approx 1.18421$ by Kumchev [8]. The admissible range for $c$ provides a measure of our progress towards the quadratic case, and it is interesting to extend it further.

We shall establish the following result.

Theorem 1. The inequality (1.2) is true for $1<c \leq \frac{243}{205} \approx 1.18536$ and $x \geq x_{0}(c)$. 
We shall prove Theorem 1 by sieving the following two sequences

$$
\mathcal{A}:=\left\{m: m=\left[n^{c}\right], X \leq n^{c}<2 X\right\}, \mathcal{B}:=\{n: X \leq n<2 X\}
$$

For this it is necessary to present some standard notations in the sieve. For any finite set $\mathcal{E} \subset \mathbb{N}, d \in \mathbb{N}$ and $z \geq 2$, we define $\mathcal{E}_{d}:=\{n \in \mathcal{E}: n \equiv 0(\bmod d)\}$ and $S(\mathcal{E}, z):=$ $|\{n \in \mathcal{E}: p \mid n \Rightarrow p \geq z\}|$. In order to prove Theorem 1, it suffices to show that

$$
S\left(\mathcal{A},(2 X)^{1 / 2}\right) \gg \frac{\delta X}{\log X} \quad\left(X \geq X_{0}\right),
$$

where and in the sequel we put $\gamma:=1 / c$ and $\delta:=X^{\gamma-1}\left(2^{\gamma}-1\right)$.

Following Baker-Harman-Rivat [1], we shall repeatedly use the Buchstab identity

$$
S\left(\mathcal{E}, z_{1}\right)=S\left(\mathcal{E}, z_{1}\right)-\sum_{z_{2} \leq p<z_{1}} S\left(\mathcal{E}_{p}, p\right) \quad\left(z_{2} \leq z_{1}\right)
$$

together with asymptotic formulas in Lemmas 3.4 and 3.5 below. For some of them we can obtain asymptotic formulas by these Lemmas and the rest may be discarded; (see (4.7) below). In order to get such asymptotic formulas, we must treat the following exponential sums:

$$
\begin{aligned}
S_{I} & :=\sum_{1 \leq h \leq H}\left|\sum_{m \sim M} \sum_{n \sim N} b_{n} e\left(h(m n)^{\gamma}\right)\right|, \\
S_{I I} & :=\sum_{1 \leq h \leq H}\left|\sum_{m \sim M} \sum_{n \sim N} a_{m} b_{n} e\left(h(m n)^{\gamma}\right)\right|,
\end{aligned}
$$

where $e(t):=e^{2 \pi i t},\left|a_{m}\right| \leq 1,\left|b_{n}\right| \leq 1, H=X^{1-\gamma+\varepsilon}, M N=X, \varepsilon$ is an arbitrarily small positive number and $m \sim M$ means $M<m \leq 2 M$. In all previous works ([1], [7], [8], [10]), these two sums were estimated by the method of Fouvry-Iwaniec [2] and by the method of Heath-Brown [5].

Here we shall combine the method of Rivat-Sargos [11] with a "Weyl shift" (Lemma 2.5 of [3]) to improve that of Heath-Brown [5]; (see Theorem 2 below). This allows us to extend $X^{1-\gamma+\varepsilon} \leq N \leq X^{5 \gamma-4-\varepsilon}$ of ([1], [7], [8], [10]) to $X^{1-\gamma+\varepsilon} \leq N \leq X^{(61 \gamma-49) / 11-\varepsilon}$, which is the key to proving Theorem 1. In addition the work of Sargos and $\mathrm{Wu}[\mathbf{1 2}$, Theorem 7] concerning the method of Fouvry-Iwaniec enables us to extend the range $\frac{16}{19}<\gamma<1$ in Lemmas 3 and 4 of [8] to $\frac{37}{44}<\gamma<1$. Finally we shall obtain (see (4.16) below)

$$
S\left(\mathcal{A},(2 X)^{1 / 2}\right) \geq\{F(\gamma)+O(\varepsilon)\} \frac{\delta X}{\log X},
$$

where $F(\gamma)$ is a very complicated function (for the definition of $F(\gamma)$, see (4.17) below). If we use $\gamma_{0}$ to denote the zero of $F(\gamma)$, then $c_{0}:=1 / \gamma_{0}$ will be our desired value. It seems quite tedious to find the exact value of $\gamma_{0}$, but using Maple we have been able to obtain a good approximation guaranteed by a sufficient precision. We shall provide the details of these numerical computations in a separate section. 
Throughout the paper (except for $\S 2$ ), we suppose that $\frac{45}{38}<c \leq \frac{243}{205}$ and write $\gamma:=1 / c$. We use $\varepsilon$ to denote an arbitrarily small positive number and put $\eta:=\varepsilon^{2}$. Finally we define $\mathcal{L}:=\log X$. The notations of $\S 2$ are independent of other sections.

2. Estimates on exponential sums. This section is devoted to investigating multiple exponential sums. The notations of this section are independent. Firstly we combine the method of Rivat-Sargos [11] with a "Weyl shift" [3, Lemma 2.5] to treat the following triple exponential sum of type II:

$$
S:=\sum_{h \sim H} \sum_{m \sim M} \sum_{n \sim N} a_{h} b_{m, n} e\left(X \frac{h^{\alpha} m^{\beta} n^{\gamma}}{H^{\alpha} M^{\beta} N^{\gamma}}\right) .
$$

TheOREM 2. Let $k \geq 2$ and $K:=2^{k+1}-2$. Let $\alpha, \beta, \gamma \in \mathbb{R}$ with $\alpha(\alpha-1)$ $\beta \gamma(\gamma-1) \neq 0$ and $(\gamma-1) /(1-\alpha) \neq 1, \cdots, k$. Let $X>0, \quad H \geq 1, \quad M \geq 1, N \geq 1$, $\mathcal{L}:=\log (2+X H M N),\left|a_{h}\right| \leq 1$ and $\left|b_{m, n}\right| \leq 1$. Let $S$ be defined as in (2.1). If $X \leq H^{2}$, then

$$
\begin{aligned}
S \ll & \left\{\left(X^{K} H^{4 K-2 k+2} M^{5 K-k} N^{5 K-3 k}\right)^{1 /(6 K-2 k)}+\left(X^{2} H^{6} M^{7} N^{9}\right)^{1 / 10}\right. \\
& \left.+\left(X H^{2} M^{4} N^{2}\right)^{1 / 4}+H(M N)^{1 / 2}+H^{3 / 4} M N+X^{-1 / 2} H M N\right\} \mathcal{L} .
\end{aligned}
$$

Proof. In view of Theorem 2 in [2], we can suppose that $X \geq M N$. Let $c_{0}, c_{1}, \cdots$ be some suitable constants depending on $\alpha, \beta, \gamma$ at most and $\Xi:=c_{0} M^{\beta} N^{\gamma}$. Let $Q \geq 10$ be a parameter to be chosen later. By the Cauchy inequality and by Lemma 5 in [11] (or [13, Lemma 2.5]) with $x_{i}=m^{\beta} n^{\gamma} / \Xi$ and $z_{i}=b_{m, n} e\left(X h^{\alpha} m^{\beta} n^{\gamma} / H^{\alpha} M^{\beta} N^{\gamma}\right)$, we have

$$
\begin{aligned}
|S|^{2} & \ll H \sum_{h \sim H}\left|\sum_{m \sim M} \sum_{n \sim N} b_{m, n} e\left(X \frac{h^{\alpha} m^{\beta} n^{\gamma}}{H^{\alpha} M^{\beta} N^{\gamma}}\right)\right|^{2} \\
& \ll H Q \sum_{m \sim M} \sum_{\substack{\tilde{m} \sim M \\
|u| \leq \Xi / Q}} \sum_{n \sim N} \sum_{\tilde{n} \sim N}\left|\sum_{h \sim H} e\left(\frac{X h^{\alpha} u}{H^{\alpha} M^{\beta} N^{\gamma}}\right)\right|,
\end{aligned}
$$

where $u=u_{\mathbf{m}}(\mathbf{n}):=m^{\beta} n^{\gamma}-\tilde{m}^{\beta} \tilde{n}^{\gamma}, \mathbf{m}:=(m, \tilde{m})$ and $\mathbf{n}:=(n, \tilde{n})$.

According to Lemma 1 in [2], the contribution of $|u| \leq \Xi \mathcal{L} / M N$ is $O\left(H^{2} M N Q \mathcal{L}\right)$. Thus after a splitting-up argument, we find, for some $\Delta \in(L / M N, 1 / Q]$, that

$$
|S|^{2} \ll H Q \mathcal{L} \sum_{m \sim M} \sum_{\substack{\tilde{m} \sim M \\ \Delta<|u| / \Xi \leq 2 \Delta}} \sum_{\substack{n \sim N \\ \tilde{n} \sim N}}\left|\sum_{h \sim H} e\left(\frac{X h^{\alpha} u}{H^{\alpha} M^{\beta} N^{\gamma}}\right)\right|+H^{2} M N Q \mathcal{L} .
$$

If $H^{\prime}:=X \Delta / H \leq \varepsilon$, by Theorem 2.1 in [3] and Lemma 1 in [2], the first term on the right-hand side of $(2.2)$ is $\ll X^{-1}(H M N)^{2} Q \mathcal{L} \ll H^{2} M N Q \mathcal{L}$. Next we suppose that $H^{\prime} \geq \varepsilon$. By applying Lemma 2.2 in [12], the innermost sum of (2.2) is equal to

$$
(X \Delta)^{-1 / 2} H \tilde{b}_{u} \sum_{h^{\prime} \in I^{\prime}} \tilde{a}_{h^{\prime}} e\left(f\left(h^{\prime}\right)\right)+O\left(\mathcal{L}+\Psi_{1}(u)+\Psi_{2}(u)\right),
$$


where $\Psi_{i}(u):=\min \left\{(X \Delta)^{-1 / 2} H, 1 /\left\|c_{i} X u / H \Xi\right\|\right\}, \quad\|t\|:=\min _{n \in \mathbb{Z}}|t-n|, \quad\left|\tilde{a}_{h^{\prime}}\right| \leq 1$, $\left|\tilde{b}_{u}\right| \leq 1, f\left(h^{\prime}\right):=c_{3} X \Delta(u / \Delta \Xi)^{1 /(1-\alpha)}\left(h^{\prime} / H^{\prime}\right)^{\alpha /(\alpha-1)}$ and $I^{\prime}$ is a subinterval of $\left[H^{\prime}, 2 H^{\prime}\right]$. Inserting into (2.2), we deduce, for some $\Delta \in(\mathcal{L} / M N, 1 / Q]$ and some $h^{\prime} \asymp H^{\prime}$, the following inequality:

$$
|S|^{2} \ll(X \Delta)^{-1 / 2} H^{2} Q S(\Delta)+H Q\left\{T_{1}(\Delta)+T_{2}(\Delta)\right\}+\left\{H^{2} M N Q+H(M N)^{2}\right\} \mathcal{L}
$$

where

$$
S(\Delta):=\sum_{m \sim M} \sum_{\substack{\tilde{m} \sim M \\
\Delta<|u| / \Xi \leq 2 \Delta}} \sum_{\substack{n \sim N \\
\tilde{n} \sim N}}\left|\sum_{h^{\prime} \in I^{\prime}} \tilde{a}_{h^{\prime}} e\left(f\left(h^{\prime}\right)\right)\right|, T_{i}(\Delta):=\sum_{\substack { m \sim M \\
\begin{subarray}{c}{\tilde{m} \sim M \\
\Delta<|u| / \Xi \leq 2 \Delta{ m \sim M \\
\begin{subarray} { c } { \tilde { m } \sim M \\
\Delta < | u | / \Xi \leq 2 \Delta } }\end{subarray}} \sum_{\substack{n \sim N \\
\tilde{n} \sim N}} \Psi_{i}(u) .
$$

According to (2.13) in [13], we have

$$
T_{i}(\Delta) \ll\left\{X^{1 / 2} \Delta M^{2} N+X^{1 / 2} M N+\Delta(M N)^{2}\right\} \mathcal{L}^{2} .
$$

Next we shall apply the method of $[\mathbf{1 1}]$ to treat $S(\Delta)$. For this we must smooth the coefficient of $u$. By the Cauchy inequality and a "Weyl shift" [3, Lemma 2.5], we have, for any $R \in\left(0, \varepsilon H^{\prime}\right]$, that

$$
|S(\Delta)|^{2} \ll\left(\Delta H^{\prime}\right)^{2}(M N)^{4} R^{-1}+\Delta H^{\prime}(M N)^{2} R^{-1} \sum_{1 \leq r \leq R} \sum_{h^{\prime} \sim H^{\prime}}\left|S_{h^{\prime}, r}(\Delta)\right|,
$$

where

$$
S_{h^{\prime}, r}(\Delta):=\sum_{m \sim M} \sum_{\substack{\tilde{m} \sim M \\ \Delta<|u| / \Xi \leq 2 \Delta}} \sum_{\substack{n \sim N \\ \tilde{n} \sim N}} e(g(u))
$$

$g(u):=Y u^{1 /(1-\alpha)}$ and $Y:=c_{3} X \Delta\left\{\left(h^{\prime}+r\right)^{\alpha /(\alpha-1)}-h^{\alpha /(\alpha-1)}\right\} / H^{\prime \alpha /(\alpha-1)}(\Delta \Xi)^{1 /(1-\alpha)}$.

Clearly $c_{4} \leq(m / \tilde{m})^{\beta / \gamma} \leq c_{5}$ for $m \sim M$ and $\tilde{m} \sim M$. We split the interval $I:=\left[c_{4}, c_{5}\right]$ into $J:=[|I| / \varepsilon \Delta]+1$ subintervals $I_{j}$ of length $\delta:=|I| / J \asymp \varepsilon \Delta$. For each $d \in \mathbb{N}$, we put

$$
\mathcal{M}_{j}(d):=\left\{\mathbf{m}: m \sim M, \tilde{m} \sim M,(m, \tilde{m})=d,(m / \tilde{m})^{\beta / \gamma} \in I_{j}\right\},
$$

and for each $\mathbf{m} \in \mathcal{M}_{j}(d)$ we define $\mathcal{N}(\mathbf{m}):=\{\mathbf{n}: n \sim N, \tilde{n} \sim N, \Delta<|u| / \Xi \leq 2 \Delta\}$. Thus

$$
S_{h^{\prime}, r}(\Delta)=\sum_{d \leq 2 M} \sum_{j \leq J} \sum_{\mathbf{m} \in \mathcal{M}_{j}(d)}\left|S_{h^{\prime}, r}^{(j)}(\Delta, \mathbf{m})\right|, S_{h^{\prime}, r}^{(j)}(\Delta, \mathbf{m}):=\sum_{\mathbf{n} \in \mathcal{N}(\mathbf{m})} e(g(u)) .
$$

We pick $a_{j} / b_{j} \in I_{j} \cup \mathbb{Q}$ with $b_{j}$ least and subject to $b_{j} \geq \delta^{-1 / 2}$. For $d_{j} \in \mathbb{Z}$ we define $\Gamma_{d_{j}}:=\left\{(x, y) \in \mathbb{R}^{2}: y=\left(a_{j} x-d_{j}\right) / b_{j}\right\}$. Clearly when $d_{j}$ runs over $\mathbb{Z}, \mathbb{Z}^{2} \cap \Gamma_{d_{j}}$ constitutes a partition of $\mathbb{Z}^{2}$. Hence we can write

$$
S_{h^{\prime}, r}^{(j)}(\Delta, \mathbf{m}) \ll \sum_{d_{j} \in \mathbb{Z}}\left|\sum_{\mathbf{n} \in \mathcal{N}(\mathbf{m}) \cap \Gamma_{d_{j}}} e(g(u))\right| .
$$


Let $\left(n_{0}, \tilde{n}_{0}\right)$ be the starting point of $\mathcal{N}(\mathbf{m}) \cap \Gamma_{d_{j}} \neq \emptyset$. Then $b_{j} \tilde{n}_{0}=a_{j} n_{0}-d_{j}$. Now we write the parameter equation of this straight line as follows:

$$
n=b_{j} l+n_{0}, \quad \tilde{n}=a_{j} l+\tilde{n}_{0} \quad\left(0 \leq l \ll N / b_{j} \asymp N / a_{j}\right) .
$$

Define

$$
u(t):=m^{\beta}\left(b_{j} t+n_{0}\right)^{\gamma}-\tilde{m}^{\beta}\left(a_{j} t+\tilde{n}_{0}\right)^{\gamma}, \quad F(t):=g(u(t)) .
$$

From (2.7) and Lemma 2.7 in [13], we deduce that

$$
S_{h^{\prime}, r}^{(j)}(\Delta, \mathbf{m}) \ll \begin{cases}\Delta N b_{j}\left|\sum_{l \ll N / b_{j}} e(F(l))\right| & \text { if } b_{j} \leq N, \\ \Delta N b_{j} & \text { if } b_{j}>N .\end{cases}
$$

Similarly to Lemma 8 in [11] (or [13, Lemma 2.8]), we easily prove the following result.

LEмма 2.1. If $(\gamma-1) /(1-\alpha) \neq 0,1, \cdots, k-1$, then

$$
\begin{aligned}
u(t) & =A\left(b_{j} t+n_{0}\right)^{\gamma-1}\{1+O(\varepsilon+\Delta)\}, \\
F^{(i)}(t) & =B\left(b_{j} t+n_{0}\right)^{(\gamma-1) /(1-\alpha)-k} b_{j}^{k}\left\{1+O_{k}(\varepsilon+\Delta)\right\} \quad(0 \leq i \leq k),
\end{aligned}
$$

where

$$
\begin{aligned}
& A:=\gamma \tilde{m}^{\beta}\left(a_{j} / b_{j}\right)^{\gamma-1}\left(d_{j} / b_{j}\right) \asymp \Delta M^{\beta} N, \\
& B:=\prod_{i=0}^{k-1}((\gamma-1) /(1-\alpha)-i) Y A^{1 /(1-\alpha)} \asymp H r N^{-(\gamma-1) /(1-\alpha)} .
\end{aligned}
$$

Applying Theorem 2.9* in [3] to the sum over $l$ in (2.8) with $(F, N)=\left(H r, N / b_{j}\right)$ yields

$$
S_{h^{\prime}, r}^{(j)}(\Delta, \mathbf{m}) \ll \Delta N^{2} \Theta_{j},
$$

where

$$
\Theta_{j}:= \begin{cases}\min \left\{1, \Theta\left(b_{j}\right)\right\} & \text { if } b_{j} \leq N, \\ b_{j} / N & \text { if } b_{j}>N,\end{cases}
$$

and $\Theta\left(b_{j}\right):=\left(H r N^{-} k b_{j}^{k}\right)^{2 /(K-2)}$. Inserting into (2.6) leads to

$$
S_{h^{\prime}, r}(\Delta) \ll \Delta N^{2} \mathcal{L} \sum_{d \leq 2 M} \sum_{j \leq J, b_{j} \leq N} \sum_{\mathbf{m} \in \mathcal{M}_{j}(d)} \Theta_{j}+\Delta N^{2} \mathcal{L} \sum_{d \leq 2 M} \sum_{j \leq J, b_{j}>N} \sum_{\mathbf{m} \in \mathcal{M}_{j}(d)} \Theta_{j} .
$$

By using Lemma 2.6 in [13], we have 


$$
\sum_{j \leq J, b_{j}>N} \Theta_{j} \ll \sum_{0 \leq i \ll \mathcal{L}} \sum_{j \leq J, 1 \leq b_{j} / 2^{i} N \leq 2} b_{j} / N \ll \sum_{0 \leq i \ll \mathcal{L}} 2^{-i}(N \delta)^{-2} \ll(N \delta)^{-2} .
$$

In addition since the points in $\mathcal{M}_{j}(d)$ are spaced by $\gg(d / M)^{2}$, we have $\left|\mathcal{M}_{j}(d)\right| \ll \delta(M / d)^{2}+1$. Thus the second member on the right-hand side of (2.11) is

$$
\ll \Delta N^{2}\left(\delta M^{2}+M\right)(N \delta)^{-2} \mathcal{L} \ll\left(M^{2}+\Delta^{-1} M\right) \mathcal{L} .
$$

Similarly Lemma 2.6 in [13] allows us to deduce that

$$
\sum_{j \leq J, b_{j} \leq N} \Theta_{j} \ll \sum_{0 \leq i \ll \mathcal{L}} \sum_{j \leq J, 1 \leq b_{j} / 2^{i} \delta^{-1 / 2} \leq 2} \Theta\left(b_{j}\right) \ll \sum_{i \geq 0} 4^{-i} \delta^{-1} \Theta\left(2^{i} \delta^{-1 / 2}\right) \ll \delta^{-1} \Theta\left(\delta^{-1 / 2}\right) .
$$

Thus the first member on the right-hand side of (2.11) is

$$
\begin{aligned}
& \ll \Delta N^{2} \mathcal{L} \sum_{d \leq \sqrt{\delta} M} \sum_{j \leq J, b_{j} \leq N} \sum_{\mathbf{m} \in \mathcal{M}_{j}(d)} \Theta\left(b_{j}\right)+\Delta N^{2} \mathcal{L} \sum_{d>\sqrt{\delta} M} \sum_{j \leq J, b_{j} \leq N} \sum_{\mathbf{m} \in \mathcal{M}_{j}(d)} 1 \\
& \ll \Delta N^{2} \mathcal{L} \sum_{d \leq \sqrt{\delta} M} \delta(M / d)^{2} \sum_{j \leq J, b_{j} \leq N} \Theta\left(b_{j}\right)+\Delta N^{2} \mathcal{L} \sum_{d>\sqrt{\delta} M}(M / d)^{2} \\
& \ll \Delta(M N)^{2} \Theta\left(\delta^{-1 / 2}\right) \mathcal{L}+\Delta \delta^{-1 / 2} M N^{2} \mathcal{L} \\
& \ll \Delta(M N)^{2} \Theta\left(\Delta^{-1 / 2}\right) \mathcal{L}+\Delta^{1 / 2} M N^{2} \mathcal{L} .
\end{aligned}
$$

Inserting (2.12) and (2.13) into (2.11), we obtain

$$
S_{h^{\prime}, r}(\Delta) \ll\left\{\left(\Delta^{K-k-2} H^{2} M^{2 K-4} N^{2 K-2 k-4} r^{2}\right)^{1 /(K-2)}+\Delta^{1 / 2} M N^{2}+M^{2}+\Delta^{-1} M\right\} \mathcal{L} .
$$

Inserting into (2.5) leads to the inequality

$$
\begin{aligned}
|S(\Delta)|^{2} \ll & \left\{\left(\Delta^{2 K-k-4} H^{\prime 2 K-4} H^{2} M^{4 K-8} N^{4 K-2 k-8} R^{2}\right)^{1 /(K-2)}\right. \\
& \left.+\left(\Delta H^{\prime}\right)^{2}(M N)^{4} R^{-1}+\left(\Delta^{3} H^{\prime 4} M^{6} N^{8}\right)^{1 / 2}+\Delta H^{\prime 2} M^{4} N^{2}+H^{\prime 2} M^{3} N^{2}\right\} \mathcal{L} .
\end{aligned}
$$

Optimizing $R$ over $\left(0, \varepsilon H^{\prime}\right]$ we get

$$
\begin{aligned}
|S(\Delta)|^{2} \ll & \left\{\left(\Delta^{2 K-k} H^{\prime 2 K} H^{2} M^{4 K} N^{4 K-2 k}\right)^{1 / K}\right. \\
& \left.+\Delta^{2} H^{\prime}(M N)^{4}+\left(\Delta^{3} H^{\prime 4} M^{6} N^{8}\right)^{1 / 2}+\Delta H^{\prime 2} M^{4} N^{2}+H^{\prime 2} M^{3} N^{2}\right\} \mathcal{L} .
\end{aligned}
$$

Replacing $H^{\prime}$ by $X \Delta / H$ yields

$$
\begin{aligned}
|S(\Delta)|^{2} \ll & \left\{\left(X^{2 K} \Delta^{4 K-k} H^{-2 K+2} M^{4 K} N^{4 K-2 k}\right)^{1 / K}+X \Delta^{3} H^{-1}(M N)^{4}\right. \\
& \left.+\left(X^{4} \Delta^{7} H^{-4} M^{6} N^{8}\right)^{1 / 2}+X^{2} \Delta^{3} H^{-2} M^{4} N^{2}+X^{2} \Delta^{2} H^{-2} M^{3} N^{2}\right\} \mathcal{L} .
\end{aligned}
$$

Inserting (2.14) and (2.4) into (2.3) and using $\Delta \leq 1 / Q$, we have

$$
\begin{aligned}
|S|^{2} \ll & \left\{\left(X^{K} H^{2 K+2} M^{4 K} N^{4 K-2 k} Q^{-(K-k)}\right)^{1 / 2 K}\right. \\
& +\left(H^{3} M^{4} N^{4}\right)^{1 / 2}+\left(X^{2} H^{4} M^{6} N^{8} Q^{-1}\right)^{1 / 4}+\left(X H^{2} M^{4} N^{2}\right)^{1 / 2} \\
& \left.+\left(X H^{2} M^{3} N^{2} Q\right)^{1 / 2}+\left(X H^{2} M^{2} N^{2} Q^{2}\right)^{1 / 2}+H^{2} M N Q+H(M N)^{2}\right\} \mathcal{L}^{2} .
\end{aligned}
$$


Since $X \leq H^{2}$, we have $\left(X H^{2} M^{2} N^{2} Q^{2}\right)^{1 / 2} \leq H^{2} M N Q$. If $Q \geq Q_{0}:=\max \{10$, $\left.X H^{-2} M\right\}$, then $\left(X H^{2} M^{3} N^{2} Q\right)^{1 / 2} \leq H^{2} M N Q$. Thus the previous estimation can be simplified as follows:

$$
\begin{aligned}
|S|^{2} \ll & \left\{\left(X^{K} H^{2 K+2} M^{4 K} N^{4 K-2 k} Q^{-(K-k)}\right)^{1 / 2 K}+\left(X^{2} H^{4} M^{6} N^{8} Q^{-1}\right)^{1 / 4}\right. \\
& \left.+H^{2} M N Q+H^{3 / 2} M^{2} N^{2}+X^{1 / 2} H M^{2} N+H(M N)^{2}\right\} \mathcal{L}^{2}
\end{aligned}
$$

Now optimizing $Q$ over $\left[Q_{0}, \infty\right)$ and noticing that $\left(X M^{2} N\right)^{1 / 2} \leq\left(X H^{2} M^{4} N^{2}\right)^{1 / 4}$, we obtain the required result. This completes the proof.

We also need another result on triple exponential sum of type II; see [12, Theorem 7].

Lemma 2.2. Let $\alpha, \beta, \gamma \in \mathbb{R}$ with $\alpha \beta \gamma(\alpha-1)(\alpha-2) \neq 0, X>0, H \geq 1, M \geq 1$, $N \geq 1, \mathcal{L}:=\log (2+X H M N),\left|a_{h}\right| \leq 1$ and $\left|b_{m, n}\right| \leq 1$. Let $S$ be defined as in (2.1). Then

$$
\begin{aligned}
S \ll & \left\{\left(X^{4} H^{15} M^{22} N^{22}\right)^{1 / 26}+\left(X H^{2} M^{3} N^{3}\right)^{1 / 4}\right. \\
& \left.+H^{11 / 18} M N+H(M N)^{3 / 4}+X^{-1 / 2} H M N\right\} \mathcal{L}^{2} .
\end{aligned}
$$

From Theorem 2 and Lemma 2.2, we immediately deduce the following result.

Corollary 1. Let $\frac{37}{44}<\gamma<\frac{61}{71}, H=X^{1-\gamma+4 \eta}, M N=X,\left|a_{m}\right| \leq 1$ and $\left|b_{n}\right| \leq 1$. If one of the following three conditions

(i) $X^{3-3 \gamma+\varepsilon} \leq N \leq X^{3 \gamma-2-\varepsilon}$,

(ii) $X^{1-\gamma+\varepsilon} \leq N \leq X^{(61 \gamma-49) / 11-\varepsilon}$,

(iii) $X^{(60-61 \gamma) / 11+\bar{\varepsilon}} \leq N \leq X^{\gamma-\varepsilon}$

holds, then

$$
\sum_{1 \leq h \leq H}\left|\sum_{m \sim M} \sum_{n \sim N} a_{m} b_{n} e\left(h(m n)^{\gamma}\right)\right| \ll X^{1-5 \eta}
$$

Proof. By using Lemma 2.2 with $(H, M, N)=\left(X / N, H_{0}, N\right)\left(H_{0} \leq H\right)$, we see that (2.15) holds provided $\frac{36}{43}<\gamma<1$ and $X^{3-3 \gamma+\varepsilon} \leq N \leq X^{(22 \gamma-15) / 7-\varepsilon}$. Interchanging $X / N$ and $N$, we find $X^{22(\gamma-1) / 7+\varepsilon} \leq N \leq X^{3 \gamma-2-\varepsilon}$. Since $22(\gamma-1) / 7 \leq 1 / 2 \leq(22 \gamma-15) / 7$ for $\gamma>\frac{37}{44}$, this proves (2.15) provided (i) holds and $\frac{37}{44}<\gamma<1$.

If (ii) holds and $\frac{5}{6}<\gamma<\frac{61}{71}$, we can get (2.15) by using Theorem 2 with $k=5$ and $(H, M, N)=\left(X / N, H_{0}, N\right)\left(H_{0} \leq H\right)$. Similarly if (iii) holds and $\frac{5}{6}<\gamma<\frac{61}{71}$, we can get (2.15) by using Theorem 2 with $k=5$ and $(H, M, N)=\left(N, H_{0}, X / N\right)\left(H_{0} \leq H\right)$.

We also need a result on triple exponential sums of type I [12, Theorem 8].

Lemma 2.3. Let $\alpha, \beta, \gamma \in \mathbb{R}$ with $\alpha \beta \gamma(\gamma-2)(\alpha+\gamma-1)(\alpha+2 \gamma-2) \neq 0, X>0$, $H \geq 1, M \geq 1, N \geq 1, \mathcal{L}:=\log (2+X H M N),\left|a_{h}\right| \leq 1$ and $\left|b_{m}\right| \leq 1$. Define 


$$
S:=\sum_{h \sim H} \sum_{m \sim M} \sum_{n \sim N} a_{h} b_{m} e\left(X \frac{h^{\alpha} m^{\beta} n^{\gamma}}{H^{\alpha} M^{\beta} N^{\gamma}}\right) .
$$

Then

$$
\begin{aligned}
S \ll & \left\{\left(X^{13} H^{15} M^{22} N^{4}\right)^{1 / 26}+\left(X^{2} H^{2} M^{3} N\right)^{1 / 4}+\left(X H^{4} M^{3} N\right)^{1 / 4}\right. \\
& \left.+\left(X^{9} H^{11} M^{18}\right)^{1 / 18}+H M+X^{-1} H M N\right\} \mathcal{L}^{2}
\end{aligned}
$$

COROLlaRY 2. Let $\frac{37}{44}<\gamma<\frac{28}{33}, H=X^{1-\gamma+4 \eta}, M N=X,\left|b_{n}\right| \leq 1$. If $N \leq X^{3 \gamma-2-\varepsilon}$, then

$$
\sum_{1 \leq h \leq H}\left|\sum_{m \sim M} \sum_{n \sim N} b_{n} e\left(h(m n)^{\gamma}\right)\right| \ll X^{1-5 \eta}
$$

Proof. By Lemma 2.3 with $(H, M, N)=\left(N, H_{0}, X / N\right)\left(H_{0} \leq H\right)$, we see that (2.16) holds provided $N \leq X^{(22 \gamma-13) / 11-\varepsilon}$ and $\frac{9}{11}<\gamma<\frac{28}{33}$. Combining this and Corollary 1(i) and noticing that $(22 \gamma-13) / 11>3-3 \gamma$ for $\gamma>\frac{46}{55}$, we deduce the desired result.

3. An alternative sieve. The aim of this section is to describe simply the alternative sieve of Baker-Harman-Rivat [1]. We insert new results on exponential sums in $\S 2$ into their alternative sieve. This allows us to improve all results there. Since the proof is very similar, we shall state our results. For more details see [1], [8] and [4].

Let $\frac{37}{44}<\gamma<\frac{28}{33}, \theta_{0}:=6 \gamma-5, \theta_{1}:=1-\gamma, \theta_{2}:=(61 \gamma-49) / 11, \theta_{3}:=3-3 \gamma$, $\theta_{4}:=3 \gamma-2, \theta_{5}:=(60-61 \gamma) / 11, \theta_{6}:=\gamma, \phi_{i}:=X^{\theta_{i}}$ for $i=1, \cdots, 6$ and $z:=(2 X)^{1 / 2}$. Define $I_{i}(\gamma):=\left[\phi_{2 i-1} X^{\varepsilon}, \phi_{2 i} X^{-\varepsilon}\right]$ for $i=1,2,3$.

Lemma 3.1. Let $\left|b_{n}\right| \ll X^{\eta}$ and $\frac{37}{44}<\gamma<\frac{28}{33}$. If $N \leq X^{\theta_{4}-\varepsilon}$, then

$$
\sum_{n \leq N} b_{n}\left|\mathcal{A}_{n}\right|=\delta X \sum_{n \leq N} b_{n} / n+O\left(\delta X^{1-3 \eta}\right) .
$$

Proof. We can proceed as in Lemma 6 in [1], by replacing Lemma 5 in [1] by Corollary 2.

Lemma 3.2. Let $\frac{37}{44}<\gamma<\frac{61}{71},\left|a_{m}\right| \ll X^{\eta / 2}$ and $\left|b_{n}\right| \ll X^{\eta / 2}$. If $N$ lies in one of $I_{i}(\gamma)$ $(i=1,2,3)$, then

$$
\sum_{\substack{m \sim M \\ m n \in \mathcal{A}}} \sum_{\substack{n \sim N \\ m}} a_{m} b_{n}=\sum_{\substack{m \sim M \\ m n \in \mathcal{B}}} \sum_{\substack{n \sim N \\ m}} a_{m} b_{n} \gamma(m n)^{\gamma-1}+O\left(\delta X^{1-3 \eta}\right) .
$$

Proof. We can proceed as in Lemma 7 in [1], by replacing Lemma 4 in [1] by Corollary 1.

Lemma 3.3. Suppose that $\frac{37}{44}<\gamma<\frac{28}{33}$ and $N \leq X^{\theta_{4}-\varepsilon}$. Then we have 


$$
\begin{gathered}
\text { PRIME NUMBERS OF THE FORM }\left[n^{c}\right] \\
\sum_{n \sim N} b_{n} S\left(\mathcal{A}_{n}, \phi_{0}\right)=\delta \sum_{n \sim N} b_{n} S\left(\mathcal{B}_{n}, \phi_{0}\right)\{1+O(1 / \mathcal{L})\}+O\left(\delta X^{1-2 \eta}\right),
\end{gathered}
$$

where $0 \leq b_{n} \ll X^{\eta}$ and $b_{n}=0$ unless $p \mid n \Rightarrow p \geq \phi_{0}$.

Proof. This can be proved in the same way as in Lemma 12 in [1], by using Lemmas 3.2 and 3.3 in place of Lemmas 7 and 9 in [1].

Lemma 3.4. Let $k$ be a given positive integer and let $\mathcal{D} \subset\{1, \cdots, k\}$. Assuming that $N$ lies in one of $I_{i}(\gamma)(i=1,2,3)$, then

$$
\sum_{p_{1}} \cdots \sum_{p_{k}}^{*} S\left(\mathcal{A}_{p_{1} \cdots p_{k}}, p_{1}\right)=\delta \sum_{p_{1}} \cdots \sum_{p_{k}}^{*} S\left(\mathcal{B}_{p_{1} \cdots p_{k}}, p_{1}\right)\{1+O(1 / \mathcal{L})\}+O\left(\delta X^{1-2 \eta}\right),
$$

where $*$ means that $\left(p_{1}, \cdots, p_{k}\right)$ satisfy $X^{\varepsilon} \leq p_{1}<p_{2}<\cdots<p_{k}, \prod_{j \in \mathcal{D}} p_{j} \asymp N$ together with no more than $\varepsilon^{-1}$ further conditions which take the form $R \leq \prod_{j \in \mathcal{J}} p_{j} \leq S$.

Proof. We can proceed as in Lemma 11 in [1], by replacing Lemma 7 in [1] by Lemma 3.2.

4. Decomposition of $S(\mathcal{A}, z)$. As described in $\S 1$, we repeatedly use the Buchstab identity (1.4) together with asymptotic formulas in Lemmas 3.4 and 3.5 to decompose $S(\mathcal{A}, z)$ such that the loss (i.e. the sums discarded) is as small as possible. Henceforth the letters $p, q, r, s$ always denote prime numbers.

By (1.4), we first write

$$
\begin{aligned}
S(\mathcal{A}, z)= & S\left(\mathcal{A}, \phi_{0}\right)-\sum_{\phi_{0} \leq p<\phi_{1}} S\left(\mathcal{A}_{p}, p\right)-\sum_{\phi_{1} \leq p<\phi_{2}} S\left(\mathcal{A}_{p}, p\right) \\
& -\sum_{\phi_{2} \leq p<\phi_{3}} S\left(\mathcal{A}_{p}, p\right)-\sum_{\phi_{3} \leq p<z} S\left(\mathcal{A}_{p}, p\right) \\
= & : S_{1}-S_{2}-S_{3}-S_{4}-S_{5},
\end{aligned}
$$

where $S_{1}$ can be evaluated asymptotically by Lemma 3.3 and $S_{3}, S_{5}$ by Lemma 3.4.

Applying three times (1.4) to $S_{2}$ yields

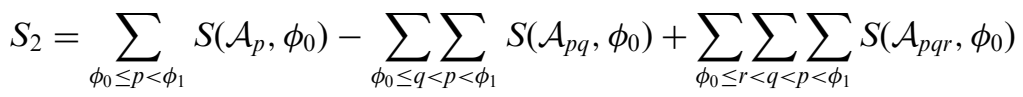

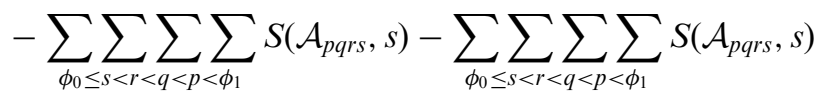

$$
\begin{aligned}
& \left(\nabla_{4}\right) \\
& \left(\nabla_{4}\right)^{\prime} \\
& =: S_{6}-S_{7}+S_{8}-S_{9}-S_{10},
\end{aligned}
$$

where

$\left(\nabla_{4}\right) \quad$ some subproducts of pqrs lie in one of the intervals $I_{i}(\gamma)(i=1,2,3)$,

$\left(\nabla_{4}\right)^{\prime} \quad$ no subproduct of pqrs lies in one of the intervals $I_{i}(\gamma)(i=1,2,3)$.

We have asymptotic formulas for $S_{6}, S_{7}, S_{8}$ by Lemma 3.3 and for $S_{9}$ by Lemma 3.4. 
In order to treat $S_{4}$ in (4.1), we first write, by (1.4),

$$
\begin{aligned}
S_{4}= & \sum_{\phi_{2} \leq p<\phi_{3}} S\left(\mathcal{A}_{p}, \phi_{0}\right)-\sum_{\phi_{2} \leq p<\phi_{3}} \sum_{\phi_{0} \leq q<\phi_{1}} S\left(\mathcal{A}_{p q}, q\right) \\
& -\sum_{\phi_{1} \leq q<\phi_{2} \leq p<\phi_{3}} S\left(\mathcal{A}_{p q}, q\right)-\sum_{\phi_{2} \leq q<p<\phi_{3}} \sum_{\phi_{1}} S\left(\mathcal{A}_{p q}, q\right) \\
= & : S_{11}-S_{12}-S_{13}-S_{14} .
\end{aligned}
$$

Lemma 3.3 provides asymptotic formulae for $S_{11}$ and Lemma 3.4 for $S_{13}$.

For $S_{12}$, we split the range of summation into three parts:

$$
\begin{aligned}
S_{12} & =\sum_{\substack{\phi_{2} \leq p<\phi_{3} \\
p q^{2}>\phi_{4}, p q \in\left[\phi_{3}, \phi_{4}\right]}} \sum_{\phi_{0} \leq q<\phi_{1}} S\left(\mathcal{A}_{p q}, q\right)+\sum_{\substack{\phi_{2} \leq p<\phi_{3} \\
p q^{2}>\phi_{4}, p q \notin\left[\phi_{3}, \phi_{4}\right]}} \sum_{\substack{\phi_{0} \leq q<\phi_{1} \\
\phi_{2} \leq p<\phi_{3} \\
p q^{2} \leq \phi_{4} \leq q<\phi_{1}}} S\left(\mathcal{A}_{p q}, q\right)+\sum_{\substack{\phi_{1}\\
}} S\left(\mathcal{A}_{p q}, q\right) \\
& =: S_{15}+S_{16}+S_{17} .
\end{aligned}
$$

According to Lemma 3.4, we have asymptotic formulas for $S_{15}$.

By applying (1.4), we have

$$
\begin{aligned}
& S_{16}=\sum_{\substack{\phi_{2} \leq p<\phi_{3} \\
p q^{2}>\phi_{4}, p q \notin\left[\phi_{3}, \phi_{4}\right]}} \sum_{\left.p q \phi_{1}\right]} S\left(\mathcal{A}_{p q},(2 X / p q)^{1 / 2}\right)+\sum_{\substack{\phi_{2} \leq p<\phi_{3} \\
p q^{2}>\phi_{4} \leq q, p q \notin\left[\phi_{3}, \phi_{4}\right],\left(\nabla_{3}\right)}} \sum_{\substack{q \\
p q)^{1 / 2}}} S\left(\mathcal{A}_{p q r}, r\right) \\
& +\sum_{\phi_{2} \leq p<\phi_{3}} \sum_{\phi_{0} \leq q<\phi_{1}} \sum_{q \leq r<(2 X / p q)^{1 / 2}} S\left(\mathcal{A}_{p q r}, r\right) \\
& p q^{2}>\phi_{4}, p q \notin\left[\phi_{3}, \phi_{4}\right],\left(\nabla_{3}\right)^{\prime} \\
& =: S_{18}+S_{19}+S_{20} \text {, }
\end{aligned}
$$

where

$\left(\nabla_{3}\right)$ some subproducts of $p q r$ lie in one of the intervals $I_{i}(\gamma)(i=1,2,3)$,

$\left(\nabla_{3}\right)^{\prime} \quad$ no subproduct of $p q r$ lies in one of the intervals $I_{i}(\gamma)(i=1,2,3)$.

We can evaluate asymptotically $S_{19}$ by Lemma 3.3 .

By applying (1.4) twice, we have

$$
\begin{aligned}
& S_{17}=\sum_{\phi_{2} \leq p<\phi_{3}} \sum_{\phi_{0} \leq q<\phi_{1}} S\left(\mathcal{A}_{p q}, \phi_{0}\right)-\sum_{\phi_{2} \leq p<\phi_{3}} \sum_{\phi_{0} \leq r<q<\phi_{1}} \sum_{p q} S\left(\mathcal{A}_{p q r}, \phi_{0}\right) \\
& p q^{2} \leq \phi_{4} \quad p q^{2} \leq \phi_{4} \\
& +\sum_{\phi_{2} \leq p<\phi_{3}} \sum_{\phi_{0} \leq s<r<q<\phi_{1}} \sum_{\phi_{1}} S\left(\mathcal{A}_{p q r s}, s\right)+\sum_{\phi_{2} \leq p<\phi_{3}} \sum_{\phi_{0} \leq s<r<q<\phi_{1}} \sum_{\phi_{4}} \sum_{\left(\mathcal{A}_{p q r s}, s\right)} \\
& p q^{2} \leq \phi_{4},\left(\nabla_{4}\right) \\
& p q^{2} \leq \phi_{4},\left(\nabla_{4}\right)^{\prime} \\
& =: S_{21}-S_{22}+S_{23}+S_{24} \text {. }
\end{aligned}
$$

Lemma 3.3 provides asymptotic formulae for $S_{21}, S_{22}$ and Lemma 3.4 for $S_{23}$.

Combining these we obtain

$$
S_{12}=S_{15}+S_{18}+S_{19}+S_{20}+S_{21}-S_{22}+S_{23}+S_{24},
$$

where $S_{15}, S_{19}, S_{21}, S_{22}, S_{23}$ can be evaluated asymptotically. 
It remains to treat $S_{14}$ in (4.3). We have

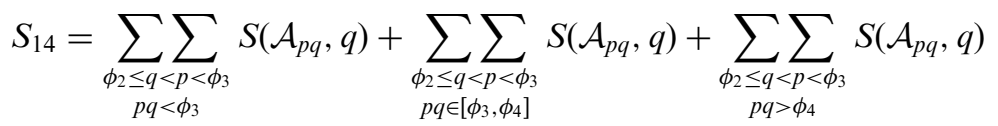

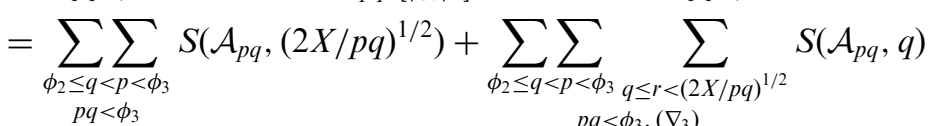

$$
\begin{aligned}
& +\sum_{\phi_{2} \leq q<p<\phi_{3}} \sum_{\substack{q \leq r<(2 X / p q)^{1 / 2} \\
p q<\phi_{3},\left(\nabla_{3}\right)^{\prime}}} S\left(\mathcal{A}_{p q r}, r\right)+\sum_{\substack{\phi_{2} \leq q<p<\phi_{3} \\
p q \in\left[\phi_{3}, \phi_{4}\right]}} S\left(\mathcal{A}_{p q}, q\right)+\sum_{\substack{\phi_{2} \leq q<p<\phi_{3} \\
p q>\phi_{4}}} \sum_{\substack{1 \\
p}} S\left(\mathcal{A}_{p q}, q\right) \\
& =: S_{25}+S_{26}+S_{27}+S_{28}+S_{29} \text {, }
\end{aligned}
$$

where $S_{26}$ and $S_{28}$ can be evaluated asymptotically by Lemma 3.4 .

Inserting (4.4) and (4.5) into (4.3) yields

$$
\begin{aligned}
S_{4}= & S_{11}-S_{15}-S_{18}-S_{19}-S_{20}-S_{21}+S_{22} \\
& -S_{23}-S_{24}-S_{13}-S_{25}-S_{26}-S_{27}-S_{28}-S_{29},
\end{aligned}
$$

where $S_{j}$ can be evaluated asymptotically, except for $j=18,20,24,25,27,29$.

Finally from (4.1), (4.2) and (4.6), we deduce that

$$
\begin{aligned}
S(\mathcal{A}, z)= & S_{1}-S_{6}+S_{7}-S_{8}+S_{9}+S_{10}-S_{3}-S_{11}+S_{15}+S_{18}+S_{19}+S_{20} \\
& +S_{21}-S_{22}+S_{23}+S_{24}+S_{13}+S_{25}+S_{26}+S_{27}+S_{28}+S_{29}-S_{5},
\end{aligned}
$$

where $S_{j}$ can be evaluated asymptotically, except for $j=10,18,20,24,25,27,29$.

Obviously the same decomposition also holds for $S(\mathcal{B}, z)$; that is

$$
\begin{aligned}
S(\mathcal{B}, z)= & S_{1}^{\prime}-S_{6}^{\prime}+S_{7}^{\prime}-S_{8}^{\prime}+S_{9}^{\prime}+S_{10}^{\prime}-S_{3}^{\prime}-S_{11}^{\prime}+S_{15}^{\prime}+S_{18}^{\prime}+S_{19}^{\prime}+S_{20}^{\prime} \\
& +S_{21}^{\prime}-S_{22}^{\prime}+S_{23}^{\prime}+S_{24}^{\prime}+S_{13}^{\prime}+S_{25}^{\prime}+S_{26}^{\prime}+S_{27}^{\prime}+S_{28}^{\prime}+S_{29}^{\prime}-S_{5}^{\prime},
\end{aligned}
$$

where $S_{j}^{\prime}$ is defined similarly to $S_{j}$ with the only difference that $\mathcal{A}$ is replaced by $\mathcal{B}$. Since $S_{j}=\delta S_{j}^{\prime}\{1+O(\varepsilon)\}$ except for $j=10,18,20,24,25,27,29$, we can obtain

$$
\begin{aligned}
S(\mathcal{A}, z)= & \delta\left\{S(\mathcal{B}, z)-S_{10}^{\prime}-S_{18}^{\prime}-S_{20}^{\prime}-S_{24}^{\prime}-S_{25}^{\prime}-S_{27}^{\prime}-S_{29}^{\prime}\right\}\{1+O(\varepsilon)\} \\
& +S_{10}+S_{18}+S_{20}+S_{24}+S_{25}+S_{27}+S_{29},
\end{aligned}
$$

from which we deduce, in view of $S_{j} \geq 0$ for $j=10,18,20,24,25,27,29$,

$$
S(\mathcal{A}, z) \geq \delta\left\{S(\mathcal{B}, z)-S_{10}^{\prime}-S_{18}^{\prime}-S_{20}^{\prime}-S_{24}^{\prime}-S_{25}^{\prime}-S_{27}^{\prime}-S_{29}^{\prime}\right\}\{1+O(\varepsilon)\} .
$$

By the prime number theorem, we have

$$
S(\mathcal{B}, z) \sim \frac{X}{\log X} .
$$

Next we shall give upper bounds for $S_{j}^{\prime}$ for $j=10,18,20,24,25,27,29$. Define $\alpha_{j}$ by the relations $p:=X^{\alpha_{1}}, q:=X^{\alpha_{2}}, r:=X^{\alpha_{3}}$ and $s:=X^{\alpha_{4}}$. Let $\omega(t)$ be the Buchstab function. 
A. Estimation of $S_{10}^{\prime}$.

According to Lemma 5 in [8], we deduce that

$$
S_{10}^{\prime}=\{1+O(\varepsilon)\} \sum_{\phi_{0} \leq s<r<q<p<\phi_{1}} \sum_{p q} \sum_{p r s \log s} \omega\left(\frac{\log (X / p q r s)}{\log s}\right) .
$$

$\left(\nabla_{4}\right)^{\prime}$

By using the standard procedure for replacing sums over primes by integrals, we can prove that

$$
S_{10}^{\prime}=\frac{X}{\log X} \int_{\mathbb{D}_{10}} \omega\left(\frac{1-\alpha_{1}-\cdots-\alpha_{4}}{\alpha_{4}}\right) \frac{d \alpha_{1} d \alpha_{2} d \alpha_{3} d \alpha_{4}}{\alpha_{1} \alpha_{2} \alpha_{3} \alpha_{4}^{2}}\{1+O(\varepsilon)\}
$$

where

$$
\mathbb{D}_{10}:=\left\{\left(\alpha_{1}, \alpha_{2}, \alpha_{3}, \alpha_{4}\right): \theta_{0} \leq \alpha_{4} \leq \alpha_{3} \leq \alpha_{2} \leq \alpha_{1} \leq \theta_{1},(C)_{4}\right\}
$$

and

$(C)_{4} \quad$ there is no subsum of $\alpha_{1}+\alpha_{2}+\alpha_{3}+\alpha_{4}$ in $\left[\theta_{1}, \theta_{2}\right],\left[\theta_{3}, \theta_{4}\right]$.

Since $\omega(t)<0.5672$ for $t \geq 2$ and $\left(1-\alpha_{1}-\cdots-\alpha_{4}\right) / \alpha_{4} \geq 2$ for $\left(\alpha_{1}, \alpha_{2}, \alpha_{3}, \alpha_{4}\right) \in \mathbb{D}_{10}$, we find that

$$
S_{10}^{\prime} \leq f_{10}(\gamma) \frac{X}{\log X}
$$

where

$$
f_{10}(\gamma):=0.5672 \int_{\mathbb{D}_{10}} \frac{d \alpha_{1} d \alpha_{2} d \alpha_{3} d \alpha_{4}}{\alpha_{1} \alpha_{2} \alpha_{3} \alpha_{4}^{2}}
$$

B. Estimation of $S_{18}^{\prime}$.

According to the definition of $S_{18}^{\prime}$, we have

$$
S_{18}^{\prime}=\sum_{\substack{\phi_{2} \leq p<\phi_{3} \\ p q^{2}>\phi_{4}, p q \notin\left[\phi_{3}, \phi_{4}\right]}} \sum_{\substack{ \\p / p}} \sum_{p<r \leq 2 X / p q} 1=\sum_{\substack{\phi_{2} \leq p<\phi_{3} \\ p q^{2}>\phi_{4}, p q \notin\left[\phi_{3}, \phi_{4}\right]}} \sum_{\phi_{0} \leq q<\phi_{1}} \frac{\{1+O(\varepsilon)\} X}{p q \log (X / p q)},
$$

from which we deduce that

$$
S_{18}^{\prime}=\left\{f_{18}(\gamma)+O(\varepsilon)\right\} \frac{X}{\log X},
$$

where

$$
f_{18}(\gamma):=\int_{\mathbb{D}_{18}} \frac{d \alpha_{1} d \alpha_{2}}{\alpha_{1} \alpha_{2}\left(1-\alpha_{1}-\alpha_{2}\right)}
$$


and

$\mathbb{D}_{18}:=\left\{\left(\alpha_{1}, \alpha_{2}\right): \theta_{2} \leq \alpha_{1} \leq \theta_{3}, \theta_{0} \leq \alpha_{2} \leq \theta_{1}, \alpha_{1}+2 \alpha_{2}>\theta_{4}, \alpha_{1}+\alpha_{2} \notin\left[\theta_{3}, \theta_{4}\right]\right\}$.

C. Estimation of $S_{20}^{\prime}$.

Similarly to $\mathbf{A}$, we have

$$
S_{20}^{\prime} \leq f_{20}(\gamma) \frac{X}{\log X}
$$

where

$$
\begin{gathered}
f_{20}(\gamma):=0.5672 \int_{\mathbb{D}_{20}} \frac{d \alpha_{1} d \alpha_{2} d \alpha_{3}}{\alpha_{1} \alpha_{2} \alpha_{3}^{2}}, \\
\mathbb{D}_{20}:=\left\{\left(\alpha_{1}, \alpha_{2}, \alpha_{3}\right): \theta_{2} \leq \alpha_{1} \leq \theta_{3}, \theta_{0} \leq \alpha_{2} \leq \theta_{1}, \alpha_{2} \leq \alpha_{3} \leq \frac{1}{2}\left(1-\alpha_{1}-\alpha_{2}\right),\right. \\
\left.\alpha_{1}+2 \alpha_{2} \geq \theta_{4},(C)_{3}\right\}
\end{gathered}
$$

and

$(C)_{3}$ there is no subsum of $\alpha_{1}+\alpha_{2}+\alpha_{3}$ in $\left[\theta_{1}, \theta_{2}\right],\left[\theta_{3}, \theta_{4}\right],\left[\theta_{5}, \theta_{6}\right]$.

D. Estimation of $S_{24}^{\prime}$.

Similarly to $\mathbf{A}$, we have

$$
S_{24}^{\prime} \leq f_{24}(\gamma) \frac{X}{\log X}
$$

where

$$
\begin{gathered}
f_{24}(\gamma):=0.5672 \int_{\mathbb{D}_{24}} \frac{d \alpha_{1} d \alpha_{2} d \alpha_{3} d \alpha_{4}}{\alpha_{1} \alpha_{2} \alpha_{3} \alpha_{4}^{2}} \\
\mathbb{D}_{24}:=\left\{\left(\alpha_{1}, \alpha_{2}, \alpha_{3}, \alpha_{4}\right): \theta_{2} \leq \alpha_{1} \leq \theta_{3}, \theta_{0} \leq \alpha_{4} \leq \alpha_{3} \leq \alpha_{2} \leq \theta_{1}, \alpha_{1}+2 \alpha_{2} \leq \theta_{4},(C)_{4}^{\prime}\right\}
\end{gathered}
$$

and

$(C)_{4}^{\prime}$ there is no subsum of $\alpha_{1}+\alpha_{2}+\alpha_{3}+\alpha_{4}$ in $\left[\theta_{1}, \theta_{2}\right],\left[\theta_{3}, \theta_{4}\right],\left[\theta_{5}, \theta_{6}\right]$.

E. Estimation of $S_{25}^{\prime}$

Similarly to $\{\mathbf{B}\}$, we have

$$
S_{25}^{\prime} \leq\left\{f_{25}(\gamma)+O(\varepsilon)\right\} \frac{X}{\log X}
$$

where 


$$
f_{25}(\gamma):=\int_{\mathbb{D}_{25}} \frac{d \alpha_{1} d \alpha_{2}}{\alpha_{1} \alpha_{2}\left(1-\alpha_{1}-\alpha_{2}\right)}
$$

and

$$
\mathbb{D}_{25}:=\left\{\left(\alpha_{1}, \alpha_{2}\right): \theta_{2} \leq \alpha_{2} \leq \alpha_{1} \leq \theta_{3}, \alpha_{1}+\alpha_{2} \leq \theta_{3}\right\}
$$

F. Estimation of $S_{27}^{\prime}$.

Similarly to $\mathbf{B}$, we have

$$
S_{27}^{\prime} \leq\left\{f_{27}(\gamma)+O(\varepsilon)\right\} \frac{X}{\log X},
$$

where

$$
f_{27}(\gamma):=\int_{\mathbb{D}_{27}} \frac{d \alpha_{1} d \alpha_{2} d \alpha_{3}}{\alpha_{1} \alpha_{2} \alpha_{3}\left(1-\alpha_{1}-\alpha_{2}-\alpha_{3}\right)},
$$

$\mathbb{D}_{27}:=\left\{\left(\alpha_{1}, \alpha_{2}, \alpha_{3}\right): \theta_{2} \leq \alpha_{2} \leq \alpha_{1} \leq \theta_{3}, \alpha_{2} \leq \alpha_{3} \leq \frac{1}{2}\left(1-\alpha_{1}-\alpha_{2}\right), \alpha_{1}+\alpha_{2} \leq \theta_{3},(C)_{3}^{\prime}\right\}$

and

$(C)_{3}^{\prime} \quad$ there is no subsum of $\alpha_{1}+\alpha_{2}+\alpha_{3}$ in $\left[\theta_{3}, \theta_{4}\right]$.

G. Estimation of $S_{29}^{\prime}$.

Similarly to $\mathbf{A}$, we have

$$
S_{29}^{\prime} \leq\left\{f_{29}(\gamma)+O(\varepsilon)\right\} \frac{X}{\log X}
$$

where

$$
f_{29}(\gamma):=\int_{\mathbb{D}_{29}} \omega\left(\frac{1-\alpha_{1}-\alpha_{2}}{\alpha_{2}}\right) \frac{d \alpha_{1} d \alpha_{2}}{\alpha_{1} \alpha_{2}^{2}}
$$

and

$$
\mathbb{D}_{29}:=\left\{\left(\alpha_{1}, \alpha_{2}\right): \theta_{2} \leq \alpha_{2} \leq \alpha_{1} \leq \theta_{3}, \alpha_{1}+\alpha_{2} \geq \theta_{4}, \alpha_{1}+2 \alpha_{2} \leq 1\right\}
$$

Inserting (4.8)-(4.15) into (4.7), we obtain

$$
S(\mathcal{A}, z) \geq\{F(\gamma)+O(\varepsilon)\} \frac{\delta X}{\log X}
$$

where

$$
F(\gamma):=1-f_{10}(\gamma)-f_{18}(\gamma)-f_{20}(\gamma)-f_{24}(\gamma)-f_{25}(\gamma)-f_{27}(\gamma)-f_{29}(\gamma) .
$$


5. End of the proof of Theorem 1: numerical calculations. The problem of computing $\gamma_{0}$ with a sufficient precision remains to compute multiple integrals over a simplex domain with sufficient precision. In general, this is a difficult question of numerical analysis. The Monte Carlo method provides an approximation but the precision is not guaranteed. Hence we choose a safer method: symbolic integration and numerical integral in 1 variable (which is safe using Simpson or another method), using Maple. Since the use of min and max is not safe with Maple, we shall manually split the domains of integration in order to simplify them. (We have slightly enlarged them, by the way.)

We have

$$
\mathbb{D}_{10} \subset \mathbb{D}_{10}^{A} \cup \mathbb{D}_{10}^{B} \cup \mathbb{D}_{10}^{C} \cup \mathbb{D}_{10}^{D} \cup \mathbb{D}_{10}^{E}
$$

where

$$
\begin{aligned}
& \mathbb{D}_{10}^{A}:=\left\{\left(\alpha_{1}, \alpha_{2}, \alpha_{3}, \alpha_{4}\right) \in \mathbb{D}_{10}: \alpha_{3}+\alpha_{4}>\theta_{2}, \alpha_{1}+\alpha_{2}+\alpha_{3}+\alpha_{4}>\theta_{4}\right\}, \\
& \mathbb{D}_{10}^{B}:=\left\{\left(\alpha_{1}, \alpha_{2}, \alpha_{3}, \alpha_{4}\right) \in \mathbb{D}_{10}: \alpha_{3}+\alpha_{4}>\theta_{2}, \alpha_{1}+\alpha_{2}+\alpha_{3}+\alpha_{4}<\theta_{3}\right\}, \\
& \mathbb{D}_{10}^{C}:=\left\{\left(\alpha_{1}, \alpha_{2}, \alpha_{3}, \alpha_{4}\right) \in \mathbb{D}_{10}: \alpha_{3}+\alpha_{4}<\theta_{1}, \alpha_{1}+\alpha_{2}>\theta_{2}, \alpha_{2}+\alpha_{3}>\theta_{2}\right\}, \\
& \mathbb{D}_{10}^{D}:=\left\{\left(\alpha_{1}, \alpha_{2}, \alpha_{3}, \alpha_{4}\right) \in \mathbb{D}_{10}: \alpha_{3}+\alpha_{4}<\theta_{1}, \alpha_{1}+\alpha_{2}>\theta_{2}, \alpha_{2}+\alpha_{3}<\theta_{1}\right\}, \\
& \mathbb{D}_{10}^{E}:=\left\{\left(\alpha_{1}, \alpha_{2}, \alpha_{3}, \alpha_{4}\right) \in \mathbb{D}_{10}: \alpha_{3}+\alpha_{4}<\theta_{1}, \alpha_{1}+\alpha_{2}<\theta_{1}\right\} .
\end{aligned}
$$

Hence

$$
\begin{aligned}
& \mathbb{D}_{10}^{A} \subset\left\{\frac{1}{4} \theta_{4}<\alpha_{1}<\theta_{1}, \frac{1}{3}\left(\theta_{4}-\theta_{1}\right)<\alpha_{2}<\alpha_{1}, \frac{1}{2} \theta_{2}<\alpha_{3}<\alpha_{2}, \theta_{2}-\alpha_{3}<\alpha_{4}<\alpha_{3}\right\}, \\
& \mathbb{D}_{10}^{B} \subset\left\{\frac{1}{2} \theta_{2}<\alpha_{1}<\theta_{3}-\frac{3}{2} \theta_{2}, \frac{1}{2} \theta_{2}<\alpha_{2}<\theta_{3}-\theta_{2}-\alpha_{1}, \frac{1}{2} \theta_{2}<\alpha_{3}<\alpha_{2}, \theta_{2}-\alpha_{3}<\alpha_{4}<\alpha_{3}\right\}, \\
& \mathbb{D}_{10}^{C} \subset\left\{\theta_{2}-\frac{1}{2} \theta_{1}<\alpha_{1}<\theta_{1}, \theta_{2}-\theta_{1}+\theta_{0}<\alpha_{2}<\alpha_{1}, \theta_{0}<\alpha_{4}<\frac{1}{2} \theta_{1}, \alpha_{4}<\alpha_{3}<\theta_{1}-\alpha_{4}\right\}, \\
& \mathbb{D}_{10}^{D} \subset\left\{\theta_{2}-\frac{1}{2} \theta_{1}<\alpha_{1}<\theta_{1}, \frac{1}{3} \theta_{2}<\alpha_{2}<\theta_{1}-\theta_{0}, \theta_{2}-\theta_{1}<\alpha_{4}<\frac{1}{2} \theta_{1}, \alpha_{4}<\alpha_{3}<\frac{1}{2} \theta_{1}\right\}, \\
& \mathbb{D}_{10}^{E} \subset\left\{\frac{1}{3} \theta_{2}<\alpha_{1}<\theta_{1}-\frac{1}{3} \theta_{2}, \frac{1}{3} \theta_{2}<\alpha_{2}<\alpha_{1}, \theta_{2}-\theta_{1}<\alpha_{3}<\frac{1}{2} \theta_{1}, \theta_{2}-\theta_{1}<\alpha_{4}<\alpha_{3}\right\} .
\end{aligned}
$$

We have

$$
\mathbb{D}_{18}=\mathbb{D}_{18}^{A} \cup \mathbb{D}_{18}^{B} \cup \mathbb{D}_{18}^{C}
$$

where

$$
\begin{aligned}
& \mathbb{D}_{18}^{A}:=\left\{\left(\alpha_{1}, \alpha_{2}\right) \in \mathbb{D}_{18}: \alpha_{1}+\alpha_{2}<\theta_{3}, \alpha_{2}<\frac{1}{2}\left(\theta_{4}-\theta_{2}\right)\right\}, \\
& \mathbb{D}_{18}^{B}:=\left\{\left(\alpha_{1}, \alpha_{2}\right) \in \mathbb{D}_{18}: \alpha_{1}+\alpha_{2}<\theta_{3}, \alpha_{2}>\frac{1}{2}\left(\theta_{4}-\theta_{2}\right)\right\}, \\
& \mathbb{D}_{18}^{C}:=\left\{\left(\alpha_{1}, \alpha_{2}\right) \in \mathbb{D}_{18}: \alpha_{1}+\alpha_{2}>\theta_{4}\right\} .
\end{aligned}
$$

Hence we have

$$
\begin{aligned}
& \mathbb{D}_{18}^{A}=\left\{\theta_{0}<\alpha_{2}<\frac{1}{2}\left(\theta_{4}-\theta_{2}\right), \theta_{4}-2 \alpha_{2}<\alpha_{1}<\theta_{3}-\alpha_{2}\right\}, \\
& \mathbb{D}_{18}^{B}=\left\{\frac{1}{2}\left(\theta_{4}-\theta_{2}\right)<\alpha_{2}<\theta_{1}, \theta_{2}<\alpha_{1}<\theta_{3}-\alpha_{2}\right\}, \\
& \mathbb{D}_{18}^{C}=\left\{\theta_{0}<\alpha_{2}<\theta_{1}, \theta_{4}-\alpha_{2}<\alpha_{1}<\theta_{3}\right\} .
\end{aligned}
$$


We have

$$
\begin{aligned}
\mathbb{D}_{20}= & \mathbb{D}_{20}^{A} \cup \mathbb{D}_{20}^{B} \cup \mathbb{D}_{20}^{C} \cup \mathbb{D}_{20}^{D} \cup \mathbb{D}_{20}^{E} \cup \mathbb{D}_{20}^{F} \cup \mathbb{D}_{20}^{G} \cup \mathbb{D}_{20}^{H} \cup \mathbb{D}_{20}^{I} \cup \mathbb{D}_{20}^{J} \cup \mathbb{D}_{20}^{K} \cup \mathbb{D}_{20}^{L} \\
& \cup \mathbb{D}_{20}^{M} \cup \mathbb{D}_{20}^{N},
\end{aligned}
$$

where

$$
\begin{aligned}
& \mathbb{D}_{20}^{A}:=\left\{\left(\alpha_{1}, \alpha_{2}, \alpha_{3}\right) \in \mathbb{D}_{20}: \alpha_{2}+\alpha_{3}<\theta_{1}, \alpha_{1}+\alpha_{2}<\theta_{3}, \alpha_{2}<\frac{1}{3}\left(\theta_{1}-\theta_{3}+\theta_{4}\right)\right\}, \\
& \mathbb{D}_{20}^{B}:=\left\{\left(\alpha_{1}, \alpha_{2}, \alpha_{3}\right) \in \mathbb{D}_{20}: \alpha_{2}+\alpha_{3}<\theta_{1}, \alpha_{1}+\alpha_{2}<\theta_{3}, \alpha_{2}>\frac{1}{3}\left(\theta_{1}-\theta_{3}+\theta_{4}\right)\right\}, \\
& \mathbb{D}_{20}^{C}:=\left\{\left(\alpha_{1}, \alpha_{2}, \alpha_{3}\right) \in \mathbb{D}_{20}: \alpha_{2}+\alpha_{3}<\theta_{1}, \alpha_{1}+\alpha_{2}>\theta_{4}\right\}, \\
& \mathbb{D}_{20}^{D}:=\left\{\left(\alpha_{1}, \alpha_{2}, \alpha_{3}\right) \in \mathbb{D}_{20}: \alpha_{2}+\alpha_{3}>\theta_{2}, \alpha_{3}<\theta_{1}, \alpha_{1}+\alpha_{3}<\theta_{3}, \alpha_{3}<\frac{1}{3}\left(2 \theta_{2}+\theta_{3}-\theta_{4}\right)\right\}, \\
& \mathbb{D}_{20}^{E}:=\left\{\left(\alpha_{1}, \alpha_{2}, \alpha_{3}\right) \in \mathbb{D}_{20}: \alpha_{2}+\alpha_{3}>\theta_{2}, \alpha_{3}<\theta_{1}, \alpha_{1}+\alpha_{3}<\theta_{3}, \alpha_{3}>\frac{1}{3}\left(2 \theta_{2}+\theta_{3}-\theta_{4}\right)\right\}, \\
& \mathbb{D}_{20}^{F}:=\left\{\left(\alpha_{1}, \alpha_{2}, \alpha_{3}\right) \in \mathbb{D}_{20}: \alpha_{2}+\alpha_{3}>\theta_{2}, \alpha_{3}<\theta_{1}, \alpha_{1}+\alpha_{3}>\theta_{4}, \alpha_{1}+\alpha_{2}<\theta_{3}, \alpha_{2}<\frac{1}{2} \theta_{1}\right\}, \\
& \mathbb{D}_{20}^{G}:=\left\{\left(\alpha_{1}, \alpha_{2}, \alpha_{3}\right) \in \mathbb{D}_{20}: \alpha_{2}+\alpha_{3}>\theta_{2}, \alpha_{3}<\theta_{1}, \alpha_{1}+\alpha_{3}>\theta_{4}, \alpha_{1}+\alpha_{2}<\theta_{3}, \alpha_{2}>\frac{1}{2} \theta_{1}\right\}, \\
& \mathbb{D}_{20}^{H}:=\left\{\left(\alpha_{1}, \alpha_{2}, \alpha_{3}\right) \in \mathbb{D}_{20}: \alpha_{2}+\alpha_{3}>\theta_{2}, \alpha_{3}<\theta_{1}, \alpha_{1}+\alpha_{3}>\theta_{4}, \alpha_{1}+\alpha_{2}>\theta_{4}\right\}, \\
& \mathbb{D}_{20}^{I}:=\left\{\left(\alpha_{1}, \alpha_{2}, \alpha_{3}\right) \in \mathbb{D}_{20}: \alpha_{3}>\theta_{2}, \alpha_{1}+\alpha_{2}<\theta_{3}, \alpha_{1}+\alpha_{3}<\theta_{3}\right\}, \\
& \mathbb{D}_{20}^{J}:=\left\{\left(\alpha_{1}, \alpha_{2}, \alpha_{3}\right) \in \mathbb{D}_{20}: \alpha_{3}>\theta_{2}, \alpha_{1}+\alpha_{2}<\theta_{3}, \alpha_{1}+\alpha_{3}>\theta_{4}, \alpha_{2}<\frac{1}{2} \theta_{2}\right\}, \\
& \mathbb{D}_{20}^{K}:=\left\{\left(\alpha_{1}, \alpha_{2}, \alpha_{3}\right) \in \mathbb{D}_{20}: \alpha_{3}>\theta_{2}, \alpha_{1}+\alpha_{2}<\theta_{3}, \alpha_{1}+\alpha_{3}>\theta_{4}, \alpha_{2}>\frac{1}{2} \theta_{2}, \alpha_{1}<\theta_{4}-\theta_{2}\right\}, \\
& \mathbb{D}_{20}^{L}:=\left\{\left(\alpha_{1}, \alpha_{2}, \alpha_{3}\right) \in \mathbb{D}_{20}: \alpha_{3}>\theta_{2}, \alpha_{1}+\alpha_{2}<\theta_{3}, \alpha_{1}+\alpha_{3}>\theta_{4}, \alpha_{2}>\frac{1}{2} \theta_{2}, \alpha_{1}>\theta_{4}-\theta_{2}\right\}, \\
& \mathbb{D}_{20}^{M}:=\left\{\left(\alpha_{1}, \alpha_{2}, \alpha_{3}\right) \in \mathbb{D}_{20}: \alpha_{3}>\theta_{2}, \alpha_{1}+\alpha_{2}>\theta_{4}, \alpha_{1}+\alpha_{3}>\theta_{4}, \alpha_{2}<1-2 \theta_{2}-\theta_{3}\right\} \text {, } \\
& \mathbb{D}_{20}^{N}:=\left\{\left(\alpha_{1}, \alpha_{2}, \alpha_{3}\right) \in \mathbb{D}_{20}: \alpha_{3}>\theta_{2}, \alpha_{1}+\alpha_{2}>\theta_{4}, \alpha_{1}+\alpha_{3}>\theta_{4}, \alpha_{2}>1-2 \theta_{2}-\theta_{3}\right\} \text {. }
\end{aligned}
$$

\section{Hence}

$$
\begin{aligned}
& \mathbb{D}_{20}^{A} \subset\left\{\theta_{0}<\alpha_{2}<\frac{1}{3}\left(\theta_{1}-\theta_{3}+\theta_{4}\right), \alpha_{2}<\alpha_{3}<2 \alpha_{2}+\theta_{3}-\theta_{4}, \theta_{4}-2 \alpha_{2}<\alpha_{1}<\theta_{3}-\alpha_{3}\right\}, \\
& \mathbb{D}_{20}^{B} \subset\left\{\frac{1}{3}\left(\theta_{1}-\theta_{3}+\theta_{4}\right)<\alpha_{2}<\frac{1}{2} \theta_{1}, \alpha_{2}<\alpha_{3}<\theta_{1}-\alpha_{2}, \theta_{4}-2 \alpha_{2}<\alpha_{1}<\theta_{3}-\alpha_{3}\right\}, \\
& \mathbb{D}_{20}^{C} \subset\left\{\theta_{0}<\alpha_{2}<\frac{1}{2} \theta_{1}, \alpha_{2}<\alpha_{3}<\theta_{1}-\alpha_{2}, \theta_{4}-\alpha_{2}<\alpha_{1}<\theta_{3}\right\}, \\
& \mathbb{D}_{20}^{D} \subset\left\{\frac{1}{2} \theta_{2}<\alpha_{3}<\frac{1}{3}\left(2 \theta_{2}+\theta_{3}-\theta_{4}\right), \theta_{2}-\alpha_{3}<\alpha_{2}<\alpha_{3}, \theta_{4}-2 \alpha_{2}<\alpha_{1}<\theta_{3}-\alpha_{3}\right\}, \\
& \mathbb{D}_{20}^{E} \subset\left\{\frac{1}{3}\left(2 \theta_{2}+\theta_{3}-\theta_{4}\right)<\alpha_{3}<\theta_{1}, \frac{1}{2}\left(\alpha_{3}+\theta_{4}-\theta_{3}\right)<\alpha_{2}<\alpha_{3}, \theta_{4}-2 \alpha_{2}<\alpha_{1}<\theta_{3}-\alpha_{3}\right\}, \\
& \mathbb{D}_{20}^{F} \subset\left\{\theta_{0}<\alpha_{2}<\frac{1}{2} \theta_{1}, \theta_{4}-2 \alpha_{2}<\alpha_{1}<\theta_{3}-\alpha_{2}, \frac{1}{2}\left(\theta_{4}-\theta_{3}+\theta_{2}\right)<\alpha_{3}<\theta_{1}\right\}, \\
& \mathbb{D}_{20}^{G} \subset\left\{\frac{1}{2} \theta_{1}<\alpha_{2}<\theta_{1}+\theta_{3}-\theta_{4}, \theta_{4}-\theta_{1}<\alpha_{1}<\theta_{3}-\alpha_{2}, \frac{1}{2}\left(\theta_{4}-\theta_{3}+\theta_{2}\right)<\alpha_{3}<\theta_{1}\right\}, \\
& \mathbb{D}_{20}^{H} \subset\left\{\frac{1}{2} \theta_{2}<\alpha_{3}<\theta_{1}, \theta_{2}-\alpha_{3}<\alpha_{2}<\alpha_{3}, \theta_{4}-\alpha_{2}<\alpha_{1}<\theta_{3}\right\}, \\
& \mathbb{D}_{20}^{I} \subset\left\{\theta_{2}<\alpha_{1}<\theta_{3}-\theta_{2}, \frac{1}{2}\left(\theta_{4}-\alpha_{1}\right)<\alpha_{2}<\theta_{1}, \theta_{2}<\alpha_{3}<\theta_{3}-\alpha_{1}\right\}, \\
& \mathbb{D}_{20}^{J} \subset\left\{\theta_{0}<\alpha_{2}<\frac{1}{2} \theta_{2}, \theta_{4}-2 \alpha_{2}<\alpha_{1}<\theta_{3}-\alpha_{2}, \theta_{2}<\alpha_{3}<\frac{1}{2}\left(1-\alpha_{1}-\alpha_{2}\right)\right\}, \\
& \mathbb{D}_{20}^{K} \subset\left\{\frac{1}{2} \theta_{2}<\alpha_{2}<\theta_{1}, \theta_{4}-2 \alpha_{2}<\alpha_{1}<\theta_{4}-\theta_{2}, \theta_{4}-\alpha_{1}<\alpha_{3}<\frac{1}{2}\left(1-\alpha_{1}-\alpha_{2}\right)\right\}, \\
& \mathbb{D}_{20}^{L} \subset\left\{\frac{1}{2} \theta_{2}<\alpha_{2}<\theta_{1}, \theta_{4}-\theta_{2}<\alpha_{1}<\theta_{3}-\alpha_{2}, \theta_{2}<\alpha_{3}<\frac{1}{2}\left(1-\alpha_{1}-\alpha_{2}\right)\right\}, \\
& \mathbb{D}_{20}^{M} \subset\left\{\theta_{0}<\alpha_{2}<1-2 \theta_{2}-\theta_{3}, \theta_{4}-\alpha_{2}<\alpha_{1}<\theta_{3}, \theta_{2}<\alpha_{3}<\frac{1}{2}\left(1-\alpha_{1}-\alpha_{2}\right)\right\}, \\
& \mathbb{D}_{20}^{N} \subset\left\{1-2 \theta_{2}-\theta_{3}<\alpha_{2}<\theta_{1}, \theta_{4}-\alpha_{2}<\alpha_{1}<1-2 \theta_{2}-\alpha_{2}, \theta_{2}<\alpha_{3}<\frac{1}{2}\left(1-\alpha_{1}-\alpha_{2}\right)\right\} .
\end{aligned}
$$


We have

$$
\mathbb{D}_{24} \subset \mathbb{D}_{24}^{A} \cup \mathbb{D}_{24}^{B} \cup \mathbb{D}_{24}^{C} \cup \mathbb{D}_{24}^{D},
$$

where

$$
\begin{aligned}
& \mathbb{D}_{24}^{A}:=\left\{\left(\alpha_{1}, \alpha_{2}, \alpha_{3}, \alpha_{4}\right) \in \mathbb{D}_{24}: \alpha_{2}+\alpha_{3}>\theta_{2}, \alpha_{3}+\alpha_{4}>\theta_{2}\right\}, \\
& \mathbb{D}_{24}^{B}:=\left\{\left(\alpha_{1}, \alpha_{2}, \alpha_{3}, \alpha_{4}\right) \in \mathbb{D}_{24}: \alpha_{2}+\alpha_{3}>\theta_{2}, \alpha_{3}+\alpha_{4}<\theta_{1}\right\}, \\
& \mathbb{D}_{24}^{C}:=\left\{\left(\alpha_{1}, \alpha_{2}, \alpha_{3}, \alpha_{4}\right) \in \mathbb{D}_{24}: \alpha_{2}+\alpha_{3}<\theta_{1}, \alpha_{1}+\alpha_{2}+\alpha_{3}+\alpha_{4}>\theta_{4}\right\}, \\
& \mathbb{D}_{24}^{D}:=\left\{\left(\alpha_{1}, \alpha_{2}, \alpha_{3}, \alpha_{4}\right) \in \mathbb{D}_{24}: \alpha_{2}+\alpha_{3}<\theta_{1}, \alpha_{1}+\alpha_{2}+\alpha_{3}+\alpha_{4}<\theta_{3}\right\} .
\end{aligned}
$$

Hence

$$
\begin{aligned}
& \mathbb{D}_{24}^{A} \subset\left\{\frac{1}{2} \theta_{2}<\alpha_{3}<\frac{1}{2}\left(\theta_{3}-\theta_{2}\right), \alpha_{3}<\alpha_{2}<\theta_{3}-\frac{3}{2} \theta_{2}, \theta_{2}<\alpha_{1}<\theta_{3}-\theta_{2}, \frac{1}{2}\left(3 \theta_{2}-\theta_{3}\right)<\alpha_{4}<\alpha_{3}\right\}, \\
& \mathbb{D}_{24}^{B} \subset\left\{\theta_{2}<\alpha_{1}<\theta_{3}-\theta_{2}, \theta_{2}-\theta_{1}<\alpha_{4}<\frac{1}{2} \theta_{1}, \theta_{2}-\alpha_{4}<\alpha_{2}<\theta_{1}, \alpha_{4}<\alpha_{3}<\theta_{1}-\alpha_{4}\right\}, \\
& \mathbb{D}_{24}^{C} \subset\left\{\theta_{0}<\alpha_{4}<\alpha_{3}<\frac{1}{2} \theta_{1}, \frac{1}{3} \theta_{2}<\alpha_{2}<\theta_{1}-\alpha_{3}, \theta_{4}-\frac{3}{2}\left(\alpha_{2}+\alpha_{3}\right)<\alpha_{1}<\theta_{3}-\alpha_{2}-\alpha_{3}\right\}, \\
& \mathbb{D}_{24}^{D} \subset\left\{\theta_{2}-\theta_{1}<\alpha_{3}<\frac{1}{2} \theta_{1}, \frac{1}{3} \theta_{2}<\alpha_{2}<\theta_{1}-\alpha_{3}, \theta_{2}<\alpha_{1}<\theta_{3}-\theta_{2}, \theta_{2}-\theta_{1}<\alpha_{4}<\alpha_{3}\right\} .
\end{aligned}
$$

We have

$$
\mathbb{D}_{25}=\left\{\left(\alpha_{1}, \alpha_{2}\right): \theta_{2}<\alpha_{2}<\frac{1}{2} \theta_{3}, \alpha_{2}<\alpha_{1}<\theta_{3}-\alpha_{2}\right\}
$$

Also, we have

$$
\mathbb{D}_{27}=\left\{\left(\alpha_{1}, \alpha_{2}, \alpha_{3}\right): \theta_{2}<\alpha_{2}<\frac{1}{2} \theta_{3}, \alpha_{2}<\alpha_{1}<\theta_{3}-\alpha_{2}, \alpha_{2}<\alpha_{3}<\theta_{3}-\alpha_{1}\right\} .
$$

Finally

$$
\mathbb{D}_{29} \subset \mathbb{D}_{29}^{A} \cup \mathbb{D}_{29}^{B} \cup \mathbb{D}_{29}^{C} \cup \mathbb{D}_{29}^{D} \cup \mathbb{D}_{29}^{E} \cup \mathbb{D}_{29}^{F},
$$

where

$$
\begin{aligned}
& \mathbb{D}_{29}^{A}:=\left\{\left(\alpha_{1}, \alpha_{2}\right): \frac{1}{2} \theta_{4}<\alpha_{1}<\frac{1}{2}\left(3 \theta_{4}-1\right), \theta_{4}-\alpha_{1}<\alpha_{2}<\alpha_{1}\right\}, \\
& \mathbb{D}_{29}^{B}:=\left\{\left(\alpha_{1}, \alpha_{2}\right): \frac{1}{2}\left(3 \theta_{4}-1\right)<\alpha_{1}<1-3 \theta_{2}, \frac{1}{3}\left(1-\alpha_{1}\right)<\alpha_{2}<\alpha_{1}\right\}, \\
& \mathbb{D}_{29}^{C}:=\left\{\left(\alpha_{1}, \alpha_{2}\right): 1-3 \theta_{2}<\alpha_{1}<\frac{1}{3}, \theta_{2}<\alpha_{2}<\alpha_{1}\right\}, \\
& \mathbb{D}_{29}^{D}:=\left\{\left(\alpha_{1}, \alpha_{2}\right): \frac{1}{3}<\alpha_{1}<\theta_{3}, \theta_{2}<\alpha_{2}<\frac{1}{2}\left(1-\alpha_{1}\right)\right\}, \\
& \mathbb{D}_{29}^{E}:=\left\{\left(\alpha_{1}, \alpha_{2}\right): \frac{1}{2}\left(3 \theta_{4}-1\right)<\alpha_{1}<\theta_{4}-\theta_{2}, \theta_{4}-\alpha_{1}<\alpha_{2}<\frac{1}{3}\left(1-\alpha_{1}\right)\right\}, \\
& \mathbb{D}_{29}^{F}:=\left\{\left(\alpha_{1}, \alpha_{2}\right): \theta_{4}-\theta_{2}<\alpha_{1}<1-3 \theta_{2}, \theta_{2}<\alpha_{2}<\frac{1}{3}\left(1-\alpha_{1}\right)\right\} .
\end{aligned}
$$

(For the integrals on $\mathbb{D}_{29}^{E}$ and $\mathbb{D}_{29}^{F}$ we make use of $(t \omega(t))^{\prime}=\omega(t-1)$ and $\omega(t) \leq 0.5644$ for $t \geq 3$.)

Using these domains we obtain $F\left(\frac{205}{243}\right)>0.0003$. Hence it suffices to choose $\gamma \geq \frac{205}{243}$; i.e. $c \leq \frac{243}{205}$. 
AcKnowledgements. The authors are indebted to the referee for his helpful suggestions.

\section{REFERENCES}

1. R. C. Baker, G. Harman and J. Rivat, Primes of the form $\left[n^{c}\right], J$. Number Theory $\mathbf{5 0}$ (1995), 261-277.

2. E. Fouvry and H. Iwaniec, Exponential sums with monomials, J. Number Theory 33 (1989), 311-333.

3. S. W. Graham and G. Kolesnik, Van der Corput's method of exponential sums (Cambridge University Press, 1991).

4. G. Harman, On the distribution of $\alpha p$ modulo one II, Proc. London Math. Soc. (3) 72 (1996), 241-260.

5. D. R. Heath-Brown, The Pjateckiı -S̆apiro prime number theorem, J. Number Theory 16 (1983), 242-266.

6. H. Iwaniec, On the presentation of almost-primes by quadratic polynomials, Invent. Math. 47 (1978), 171-188.

7. C. H. Jia, On Piatetski-Šhapiro's prime number theorem I, Chinese Ann. Math. Ser. B 15 (1994), 9-22; II, Science in China 36 (1993), 913-926.

8. A. Kumchev, On the distribution of prime numbers of the form $\left[n^{c}\right]$, Glasgow Math. J. 41 (1999), 85-102.

9. I. I. Pjatecki1-S̆apiro, On the distribution of prime numbers in sequences of the form $[f(n)]$, Mat. Sb. 33 (1953), 559-566.

10. J. Rivat, Autour d'un théorème de Pjateckil-S̆apiro: (nombres premiers dans la suite $\left[n^{c}\right]$ ), Thèse de Doctorat (Université Paris-Sud, 1992).

11. J. Rivat and P. Sargos, Nombres premiers de la forme $\left[n^{c}\right]$, Preprint.

12. P. Sargos and J. Wu, Multiple exponential sums with monomials and their applications in number theory, Acta Math. Hungar. 87 (4) (2000), 333-354.

13. J. Wu, The square-free and cube-free divisor problems, Preprint. 